\title{
Design and Fabrication of Clock Synchronization System Based on Single Chip Microcomputer
}

\author{
Ming Tang, Yawei Li \\ School of Intelligence Science and Information Engineering \\ Xi'an Peihua University, Xi'an, China \\ 81408240@qq.com,10607702742@qq.com
}

Keywords: Clock synchronization system; Single-chip microcomputer; Serial communication; Internet of Things

\begin{abstract}
The introduction of the Internet of Things system facilitates the involvement of traditional industries, but the problem of time synchronization between the upper computer system and the lower computer system of the Internet of Things may be worth studying and solving. This paper mainly introduces a complete set of solutions for the Internet of Things clock system. The hardware system introduces the clock display and storage system based on a 51-core microcontroller; the software system introduces the Bluetooth communication data docking system for Android.The system solution uses the SCM interrupt system to send Bluetooth data. The data contains an accurate communication protocol, completes the checking of data header, data body, and data CRC16 data. The accurate synchronization of the clock system is accomplished through high-speed data communication.
\end{abstract}

\section{Introduction}

With the advanced development of science and economic, the overall performance of electronic products has been greatly optimized, making the frequency of product replacement faster and faster. The use of electronic products has taken longer and longer, and the rest time has been severely crushed. Sometimes, when using electronic products, the original plan was forgotten, adding to the people a lot of trouble.

Nowadays, in the era of rapid development of the Internet of Things, more and more emerging products emerge in an endless stream, including humanized smart home systems and unmanned warehouse management systems for large companies. The premise of normal operation of these devices is that the system can be time synchronized, in order to make the control terminal instructions effective in real time.

Due to the variety of clock synchronizers currently on the market, cumbersomeness of the set-up time steps, and multiple settings buttons need to be set in order to meet a demand, the beginners cannot quickly master the device, wasting valuable time. And when some countdown timers complete a task, the setting steps before need to be re-debugged before, which leads to users a great deal of boredom, as well as reduced time accuracy sometimes due to traditional device problems.

\section{The Clock Synchronization System}

The time information received by the system device is used to control the clock information inside the system device, that is, clock synchronization, so that the accuracy is close to the second level. The principle of clock synchronization is similar to that of frequency synchronization, but the clock synchronization system can also directly regulate the frequency of the clock and the phase change of the clock, and the phase can be expressed as a numerical value. 


\section{Hardware Design}

The system design uses the Bluetooth module to reiceve the instruction signal sent by Android mobile phone, uses the STC89C52 microcontroller to extract and process the high-accuracy time information received by the Bluetooth module, indirectly controls the electronic clock, and the electronic clock analyzes and calculates the difference between the received time information and the original time information, modify the time value and the calibration value to be consistent with the clock source accuracy to complete the clock synchronization system design.

\subsection{Clock Module Design of Clock Source Part}

The clock circuit is a basic circuit for the digital tube time counting. The main circuit is generally composed of a DS1302 clock chip and a crystal oscillator. Theoretically, in order to make the crystal frequency more accurate, two 6pf capacitors are usually added across the crystal. As shown in Fig.1.

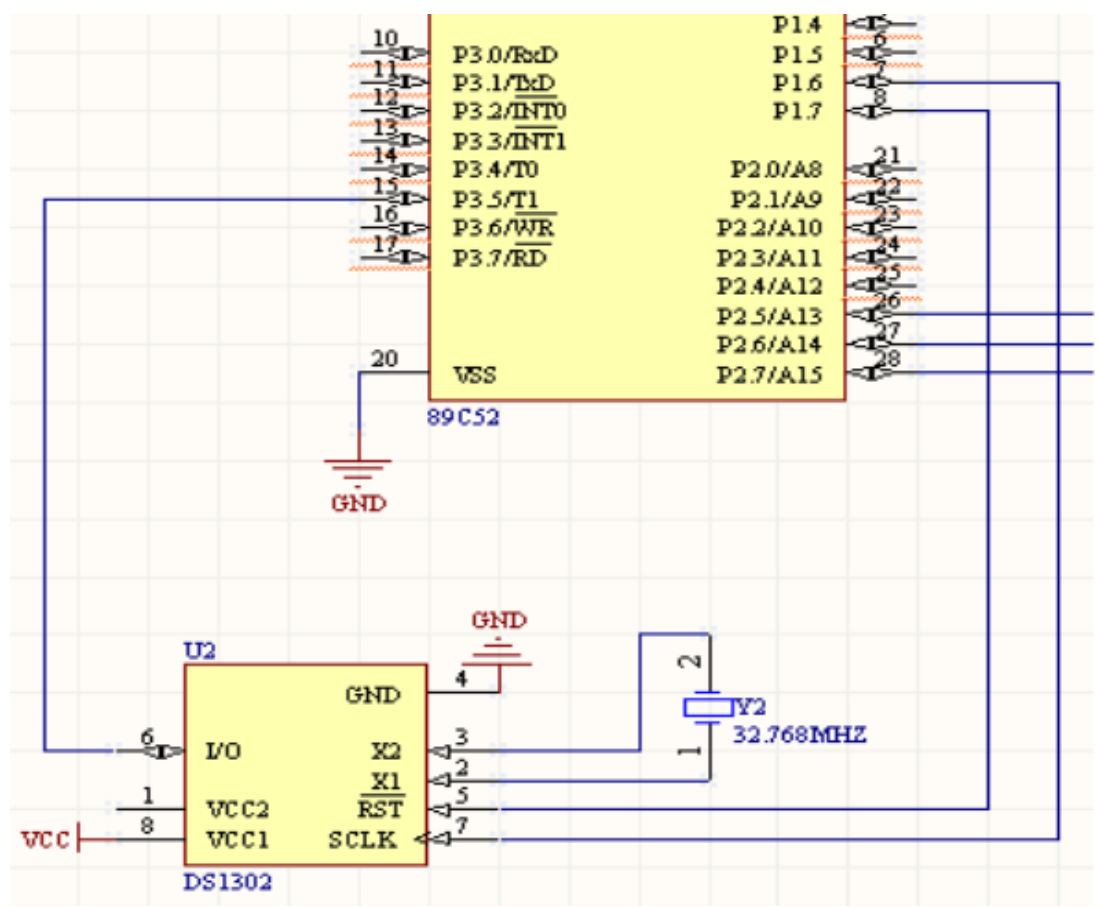

Figure 1. DS1302 clock circuit

\subsection{Digital Tube Display Module Design}

In the dynamic display, the installed digital tube will receive the signal of same segment selection, but whether to display is determined by the bit selection terminal. When the digit selection terminal of the digital tube is opened, the selected digital tube will be displayed, otherwise it is off and cannot be displayed. Only one digital tube is displayed at a time, that is, the numbers are displayed in turn during the actual operation, but as long as the rotational operation speed reaches a certain range, what the human eye sees will be the overall display effect. Because the instructions controlled by the program are different at different times, the digital control terminal selection is different, so that the dynamic display of the digital tube can be realized.

\subsection{Bluetooth Control Module Design}

The HC-06 series is used for the Bluetooth module design. Compared with the HC-05, the main advantage is that the master device can perform instruction switching. The operation is simple. It is widely used to search for slave devices, but it cannot be searched by other devices as a master device. Devices that are used to be searched, and that cannot actively search for other devices, are slave devices. After the master-slave device's connection is recognized, it can simply be seen as a serial line to transmit data, and the master-slave device's relationship boundaries will not be so obvious. As shown in Fig.3. 


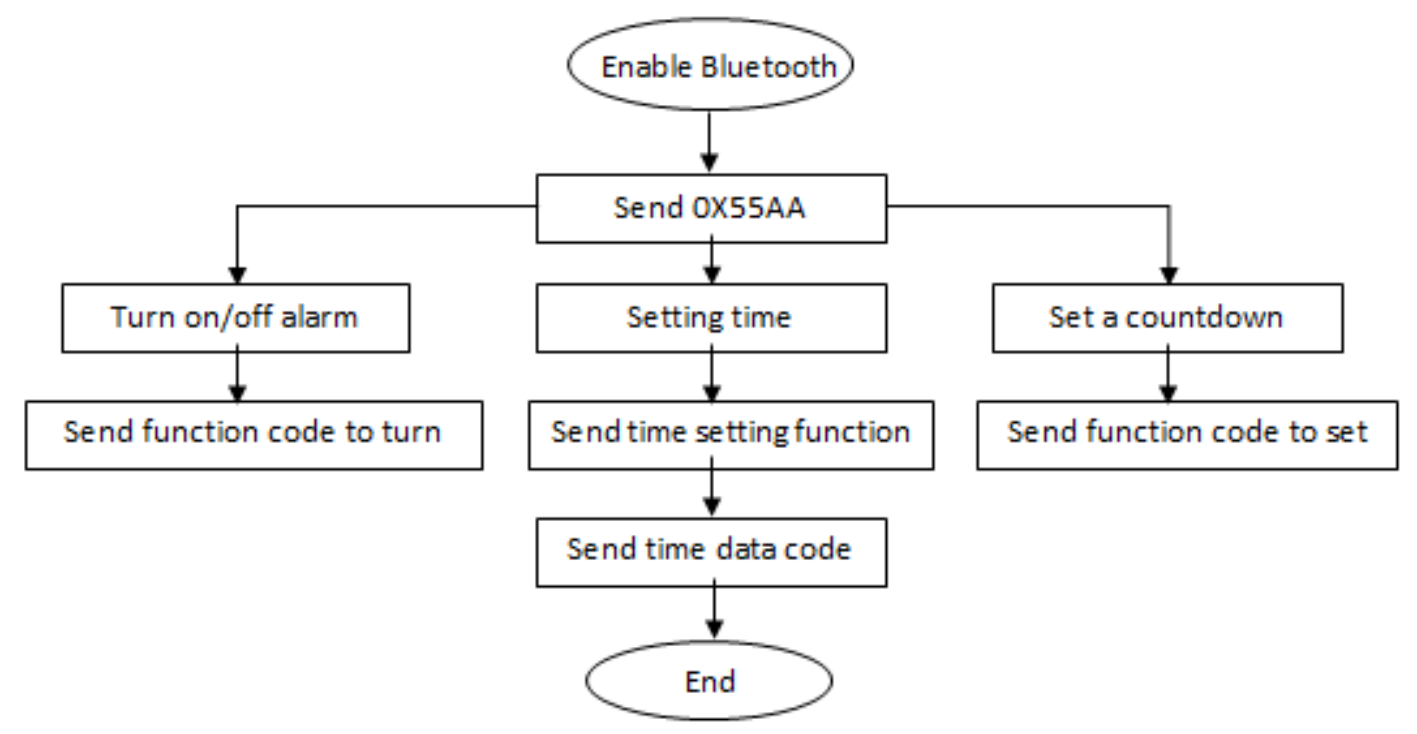

Figure 3. Bluetooth module design flow chart

\section{Software Design}

\subsection{Clock Storage and Read Drivers}

The DS1302 clock chip has the function of automatically saving time and power saving, which is cheap and suitable for mass production. The data communication of the DS1302 is different from the general communication of the SPI, but communication is not completely in accordance with the SPI communication rules. The following part will introduce the alternative SPI data communication of DS1302, as shown in Fig.4.

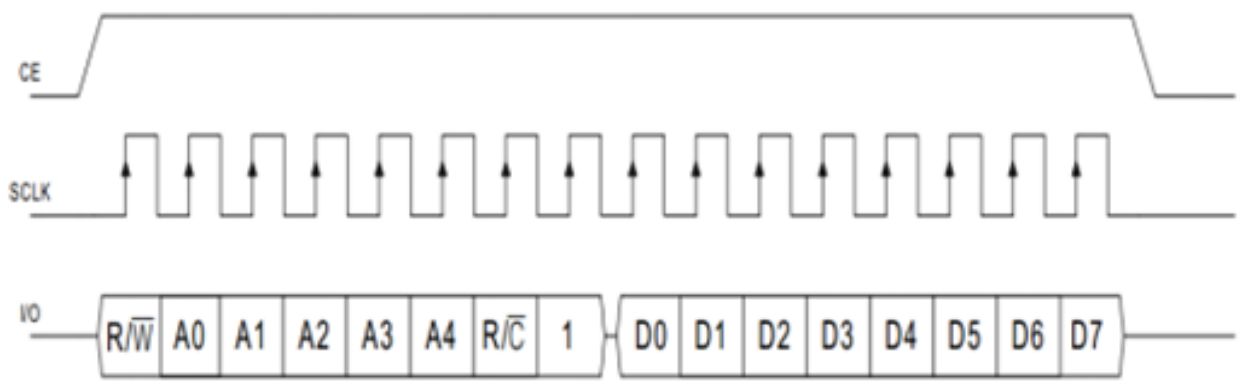

Figure 4. DS1302 Single-Byte Write Operation

Then we compare the operation sequence of SPI in the case of $\mathrm{CPOL}=0 / \mathrm{CPHA}=0$, as shown in Fig.5.

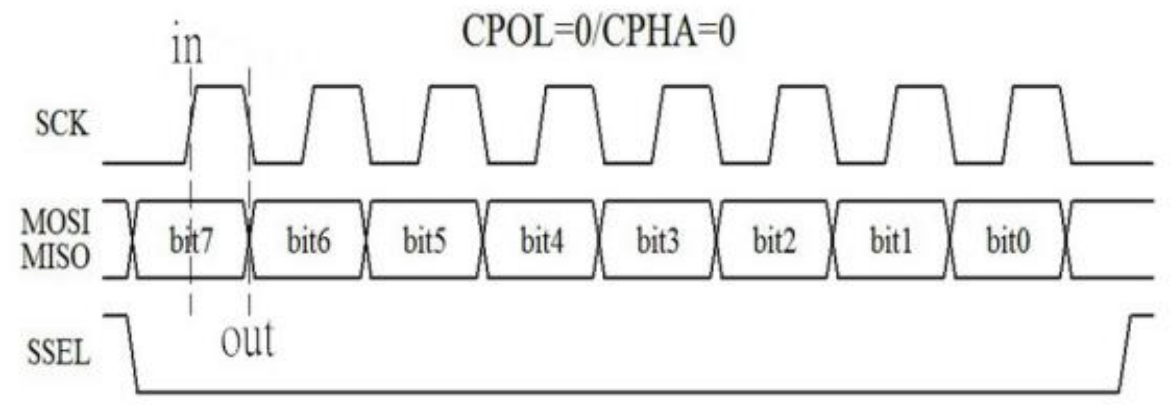

Figure 5. $\quad \mathrm{CPOL}=0 / \mathrm{CPHA}=0$ communication sequence

The communication timings of Fig.4. and Fig.5. are as shown in the figure, from which it is found that the pin enable control signals of CE and SSEL are actually the opposite. For such communication write data, they are located at positive edge of the SCK pin for sampling the data from the machine. When the negative edge of the circuit occurs, the master machine sends data. The DS1302 timing diagram is shown in Fig.6. 


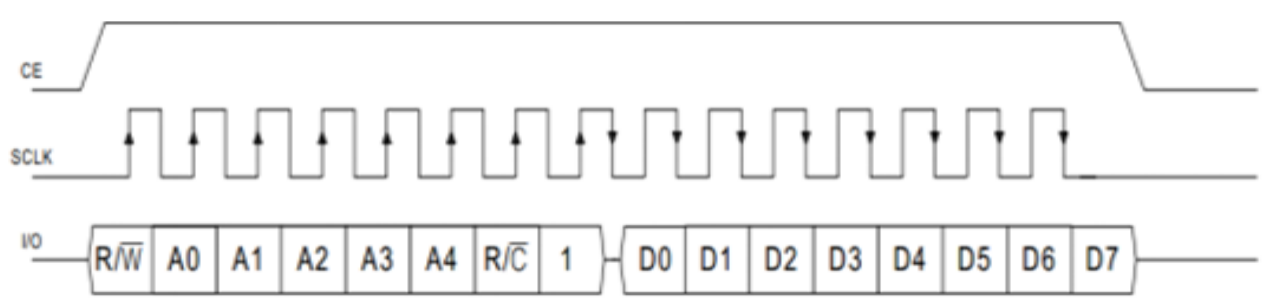

Figure 6. DS1302 Single-Byte Read Operation

There are several points that need special attention in the above reading operation. The first point is that the specific connotation of the arrows on the timing diagram of the DS1302 chip is meaningful to the DS1302 clock chip. When the read operation is completed, the first byte instruction needs to be written. When the positive edge of the timing is completed, the clock chip will be latched and the STC89C52 microcontroller will execute the transmit data at the time the sequence completes the negative edge operation. When the second data needs to be processed, the timing processing is opposite to the previous execution. The data is sent to the front edge, and the trailing edge is used to read the data.

The second point is that when using the STC89C52 microcontroller, because the C51 microcontroller internal resources do not have the standard SPI interface required for the project, like the usual communication interface, IO interface of the microcontroller is often used to simulate the corresponding communication data process when the read operation is completed. The SPI communication needs to be read with the positive edge when using the microcontroller's IO interface for simulation. There is certain time error between the data read operation and the clock edge change. Through the simulation experiment of the one-chip computer, it is found that only when the SPI interface is first read the data on the IO, a positive edge is generated when the SCLK is pulled high. At this time, the obtained data will represent a certain meaning, otherwise it is an erroneous result.

\subsection{Android Interface Development}

The system is researched and developed on an open source mobile development platform. Access to various mobile devices is the advantage of the Android development platform that is different from other development platforms.

Although the Android development platform can achieve many interesting and useful features, but sometimes in the development the XML data format is also needed, because access to data on the Internet is likely to be the XML data format, and XML can provide some unique technical support. In the various services that typically send data to the Web, there is also a great possibility of using XML. In other words, when using an Android phone application, it will use the Internet, so you can't avoid using XML. As shown in Fig.7.

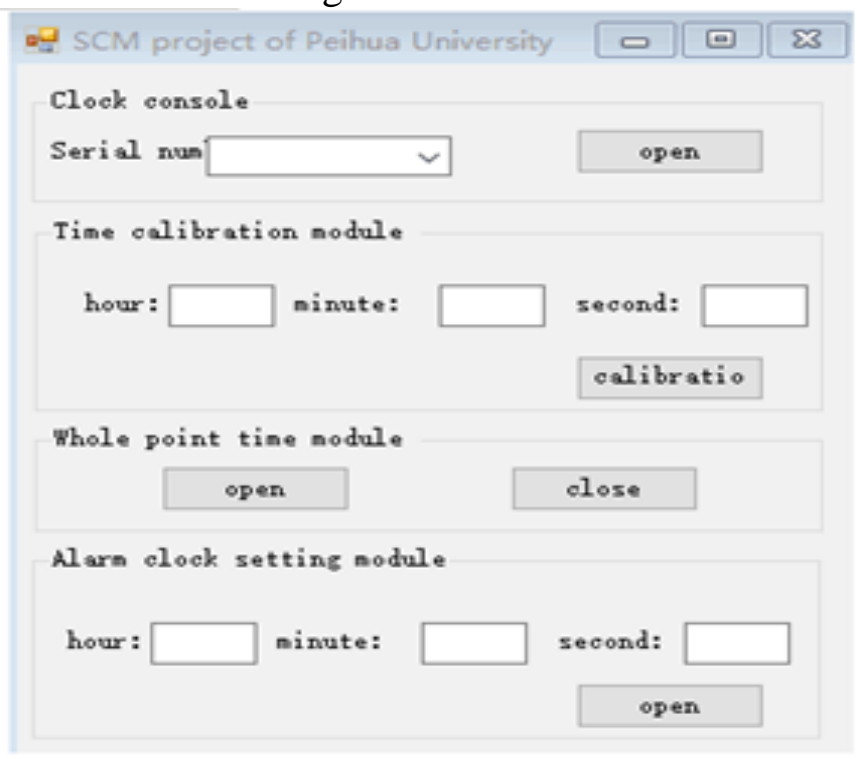

Figure 7. Android interface 
Program flow chart is shown in Fig.8.

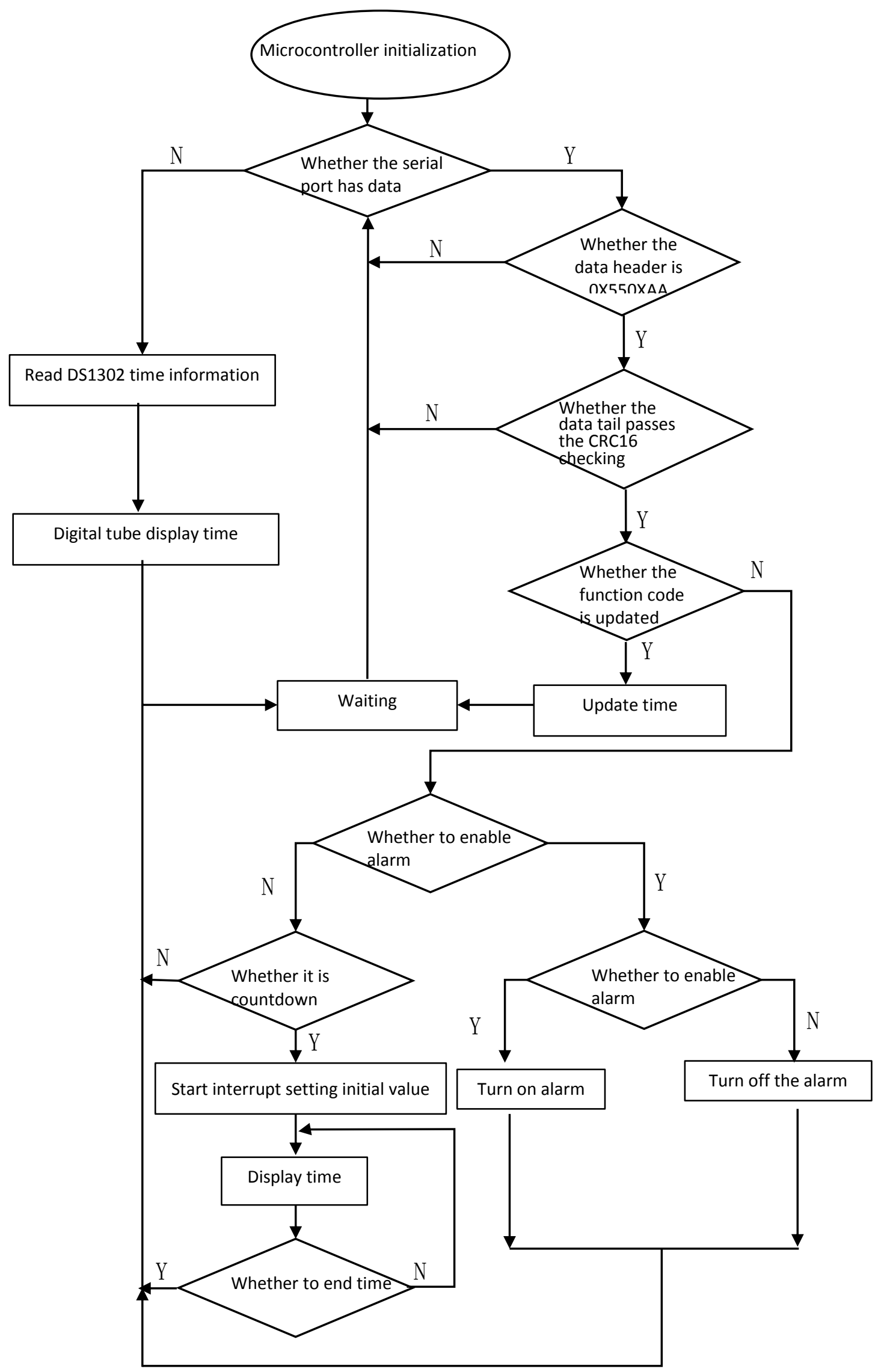

Figure 8. System flow chart 


\section{Conclusion}

This article focuses on the hardware design and software design flow of the clock synchronization system. Using the specific time information received by the Bluetooth module from the Android mobile phone terminal, enhancing the accuracy of the clock source; each clock module is directly controlled or indirectly controlled by the single chip microcomputer, and has many features different from common systems. The clock module receives the clock source from the Bluetooth transmission, and then completes the project development on the communication between the Android mobile phone APP and the single chip microcomputer based on the wireless communication technology of the Android platform.

\section{References}

[1] Louis E., Frenzel Jr (author), Ci Lin (Translation). 2017. Serial Communication Interface Specification and Standards [M]. Beijing: Tsinghua University Press.

[2] Yunbo Zhou. 2017. Internet serial communication [M]. Beijing: Electronic Industry Press.

[3] Guogang Cheng. 2016. 51 common microcontroller module design query manual [M]. Beijing: Tsinghua University Press.

[4] Yujiang Jiang. 2016. Self-designed CPU and SCM [M]. Beijing: People's Posts and Telecommunications Press.

[5] Hancai Hu. 2010. Microcontroller principle and its interface technology [M]. Beijing: Tsinghua University Press. 